\title{
Long-Range Ferromagnetic Dipolar Ordering of High-Spin Molecular Clusters
}

\author{
A. Morello, ${ }^{1}$ F. L. Mettes, ${ }^{1}$ F. Luis, ${ }^{2}$ J. F. Fernández, ${ }^{2}$ J. Krzystek, ${ }^{3}$ G. Aromí, ${ }^{4}$ G. Christou, ${ }^{5}$ and L. J. de Jongh ${ }^{1, *}$ \\ ${ }^{1}$ Kamerlingh Onnes Laboratory, Leiden Institute of Physics, Leiden University, P.O. Box 9504, 2300 RA Leiden, The Netherlands \\ ${ }^{2}$ Instituto de Ciencia de Materiales de Aragón, CSIC-Universidad de Zaragoza, 50009 Zaragoza, Spain \\ ${ }^{3}$ National High Magnetic Field Laboratory, Tallahassee, Florida 32310 \\ ${ }^{4}$ Gorlaeus Laboratories, Leiden Institute of Chemistry, Leiden University, P.O. Box 9502, 2300 RA Leiden, The Netherlands \\ ${ }^{5}$ Department of Chemistry, University of Florida, Gainesville, Florida 32611
}

(Received 19 June 2002; published 10 January 2003)

\begin{abstract}
We report the first example of a transition to long-range magnetic order in a purely dipolarly interacting molecular magnet. For the magnetic cluster compound $\mathrm{Mn}_{6} \mathrm{O}_{4} \mathrm{Br}_{4}\left(\mathrm{Et}_{2} \mathrm{dbm}\right)_{6}$, the anisotropy experienced by the total spin $S=12$ of each cluster is so small that spin-lattice relaxation remains fast down to the lowest temperatures, thus enabling dipolar order to occur within experimental times at $T_{c}=0.16 \mathrm{~K}$. In high magnetic fields, the relaxation rate becomes drastically reduced and the interplay between nuclear- and electron-spin lattice relaxation is revealed.
\end{abstract}

PACS numbers: 75.10.Jm, 75.30.Kz, 75.45.+j

Few examples of long-range magnetic order induced by purely dipolar interactions are known as yet $[1,2]$. Therefore, the possibility to study such phase transitions and the associated long-time relaxation phenomena in detail in high-spin molecular cluster compounds, with varying crystalline packing symmetries and different types of anisotropy, presents an attractive subject [3]. However, for the most extensively studied molecular clusters so far, such as $\mathrm{Mn}_{12}, \mathrm{Fe}_{8}$, and $\mathrm{Mn}_{4}$ [4-7], the uniaxial anisotropy experienced by the cluster spins is very strong. Consequently, the electronic spin-lattice relaxation time $T_{1}^{\mathrm{el}}$ becomes very long at low temperatures and the cluster spins become frozen at temperatures of the order of $1 \mathrm{~K}$, i.e., much higher than the ordering temperatures $T_{c} \sim$ $0.1 \mathrm{~K}$ expected on the basis of the intercluster dipolar couplings [3]. Although quantum tunneling of these cluster spins has been observed [4-7], and could in principle provide a relaxation path towards the magnetically ordered equilibrium state [3], the associated rates in zero field are extremely small $(<100 \mathrm{~Hz})$. For these systems, tunneling becomes effective only when strong transverse fields $B_{t}$ are applied to increase the tunneling rate. Although the tunability of this rate and thus of $T_{1}^{e l}$ by $B_{t}$ could recently be demonstrated for $\mathrm{Mn}_{12}, \mathrm{Fe}_{8}$, and $\mathrm{Mn}_{4}$ [7], no ordering has yet been observed.

The obvious way to obtain a dipolar molecular magnet is thus to look for a high-spin molecule having sufficiently weak magnetic anisotropy and negligible intercluster superexchange interactions. Here we report data for $\mathrm{Mn}_{6} \mathrm{O}_{4} \mathrm{Br}_{4}\left(\mathrm{Et}_{2} \mathrm{dbm}\right)_{6}$, hereafter abbreviated as $\mathrm{Mn}_{6}$ [8]. The molecular core of $\mathrm{Mn}_{6}$ is a highly symmetric octahedron of $\mathrm{Mn}^{3+}$ ions (with spin $s=2$ ) that are ferromagnetically coupled via strong intracluster superexchange interactions (Fig. 1). Accordingly, the ground state is a $S=12$ multiplet and the energy of the nearest excited state is approximately $150 \mathrm{~K}$ higher [8]. The unit cell is monoclinic, with space group $P c$, and contains four molecules [9] bound together only by van der Waals forces. Intercluster superexchange is therefore negligible and only dipolar interactions couple the cluster spins. The net magnetocrystalline anisotropy of this cluster proves to be sufficiently small to enable measurements of its equilibrium magnetic susceptibility and specific heat down to our lowest temperatures $(15 \mathrm{mK})$.

The main results of this paper follow. We have observed that the magnetic clusters do undergo a transition to a long-range ferromagnetically ordered state at $T_{c}=$ $0.161(2) \mathrm{K}$. This transition can be observed within experimental times (1-100 s) because the magnetic relaxation is not too slow at very low temperatures. We have obtained further information on the magnetic relaxation by applying a magnetic field. It turns out that the relaxation rate vanishes exponentially as the field increases. The interplay between nuclear- and electron-spin lattice relaxation is also revealed.

Polycrystalline samples of $\mathrm{Mn}_{6}$ were prepared as in Ref. [9]. The specific heat of a few milligrams of sample, mixed with Apiezon grease, was measured at low- $T$ in a homemade calorimeter [7] that makes use of the thermal (a)

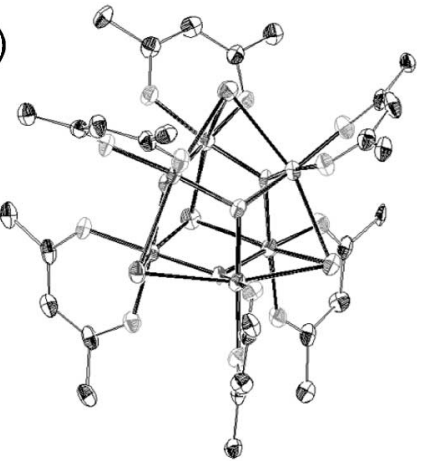

(b)

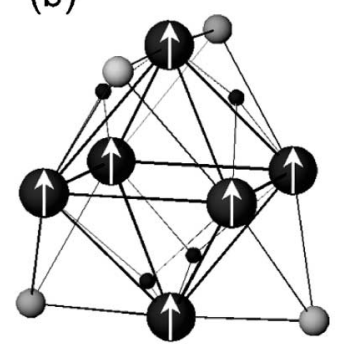

FIG. 1. (a) Crystal structure of $\mathrm{Mn}_{6} \mathrm{O}_{4} \mathrm{Br}_{4}\left(\mathrm{Et}_{2} \mathrm{dbm}\right)_{6}$ [8]; (b) detail of the symmetric octahedral core, containing six ferromagnetically coupled $\mathrm{Mn}^{3+}$ ions, yielding a total spin $S=12$ for this molecular superparamagnetic particle. 
relaxation method. An important advantage of this method is that the characteristic time $\tau_{e}$ of the experiment (typically, $\tau_{e} \simeq 1-100$ seconds at low- $T$ ) can be varied by changing the dimensions (and therefore the thermal resistance) of the Au wire that acts as a thermal link between the calorimeter and the mixing chamber of the ${ }^{3} \mathrm{He}-{ }^{4} \mathrm{He}$ dilution refrigerator. The ac susceptibility data were taken between 0.015 and $4 \mathrm{~K}$ with a mutual inductance bridge. The frequency was varied from 230 to $7700 \mathrm{~Hz}$. Magnetic data for $T>1.8 \mathrm{~K}$ were taken with a SQUID magnetometer.

We first discuss the magnitude of the anisotropy. The spin Hamiltonian for the molecule is

$$
\mathcal{H}=-D S_{z}^{2}-g \mu_{B} \vec{B} \cdot \vec{S} .
$$

Previously published magnetic data for $T>1.8 \mathrm{~K}$ already indicated an upper limit of about $0.01 \mathrm{~K}$ for $D / k_{B}$ [8]. In order to obtain an independent estimate of $D$, high-frequency ESR data were taken in the range 95-380 GHz. Owing to the combination of very small $D$ and large $S$, as well as the presence of a signal at $g=$ 2.00 arising from a minute amount of $\mathrm{Mn}^{2+}$ impurity (often seen in ESR of $\mathrm{Mn}^{3+}$ compounds), the interpretation of the spectra was not fully conclusive. Nevertheless, signals with a clearly visible structure on the low-field end of the spectra could be obtained. It could be identified as fine structure originating from zero-field splitting (ZFS), since it was independent of field and frequency. Simulations of the spectra performed using Eq. (1) agree well with the experiment taking $|D| / k_{B} \sim 0.03$ or $\sim 0.05 \mathrm{~K}$, depending on the sign of $D$ (which could not be unequivocally determined). Although a smaller rhombic component could be present, the data do not justify a more elaborate fitting.

The isotropic character of the molecular spin might seem paradoxical at first, considering that the individual $\mathrm{Mn}^{3+}$ ions, being Jahn-Teller ions, experience strong anisotropy. However, the spin Hamiltonian for the cluster is determined by the vectorial addition of the local tensors of the individual atoms, which can give rise to a low net anisotropy for highly symmetric molecules such as $\mathrm{Mn}_{6}$ (cf. Fig. 1), no matter how large the ZFS of the constituting atoms [10]. In fact, the possibility to tune the net anisotropy of the cluster spin is one of the attractive properties of molecular superparamagnets.

As first evidence for ordering of the magnetic moments we show zero-field ac susceptibility data in Fig. 2. The real part $\chi^{\prime}$ shows a sharp maximum at $T_{c}=0.161(2) \mathrm{K}$. We found that $\chi^{\prime}$ at $T_{c}$ is close to the estimated limit for a ferromagnetic powder sample, $1 /\left(\rho N_{s}+\rho_{\text {sam }} N_{c}\right) \simeq$ $0.14 \pm 0.02 \mathrm{emu} / \mathrm{g}$, where $\rho=1.45 \mathrm{~g} / \mathrm{cm}^{3}$ and $\rho_{\text {sam }} \simeq$ $0.45 \mathrm{~g} / \mathrm{cm}^{3}$ are, respectively, the densities of bulk $\mathrm{Mn}_{6}$ and of the powder sample, $N_{s}=4 \pi / 3$ is the demagnetizing factor of a crystallite, approximated by a sphere, and $N_{c} \simeq 2.51$ is the demagnetizing factor of the sample holder. This indicates that $\mathrm{Mn}_{6}$ is ferromagnetically or-

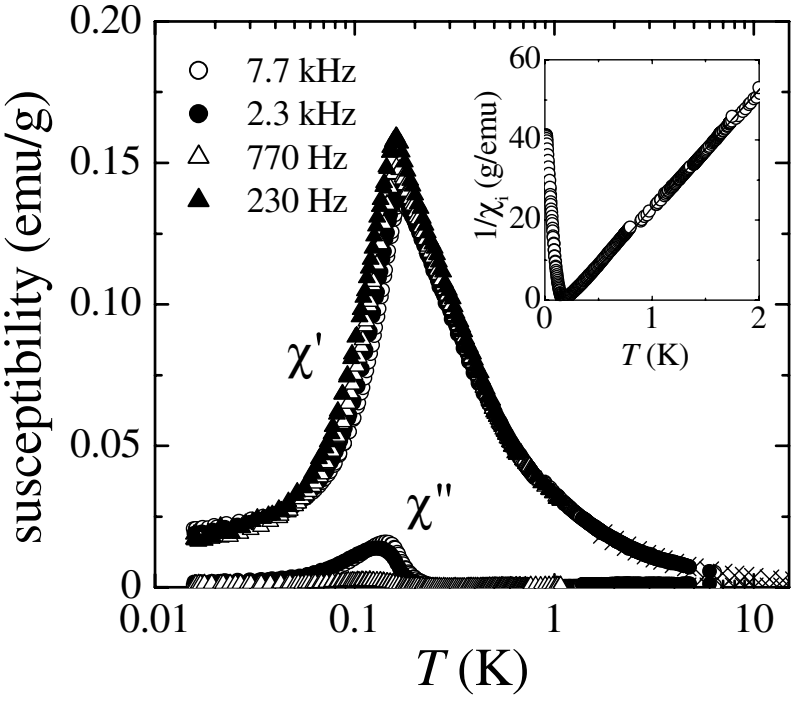

FIG. 2. The ac susceptibility of $\mathrm{Mn}_{6}$ at zero field and four different frequencies. Data measured above $1.8 \mathrm{~K}$ in a commercial SQUID magnetometer are also shown $(X)$. Inset: $\chi^{\prime}$ corrected for demagnetization effects. The full line shows the best fit of a Curie-Weiss law to the data for $T>0.3 \mathrm{~K}$, yielding $\theta=0.20(3) \mathrm{K}$.

dered below $T_{c}$. Susceptibility data $\chi_{i}$ corrected for the demagnetizing field $\left(\chi_{i}=\chi^{\prime} /\left[1-\left(\rho N_{s}+\rho_{\text {sam }} N_{c}\right) \chi^{\prime}\right]\right)$ follow the Curie-Weiss law $\chi=C /(T-\theta)$ down to approximately $0.3 \mathrm{~K}$, with $C=0.034(1) \mathrm{cm}^{3} \mathrm{~K} / \mathrm{g}$ and $\theta=$ $0.20(3) \mathrm{K}$. The constant $C$ equals, within the experimental errors, the theoretical value for randomly oriented crystals with Ising-like anisotropy $N_{A} g^{2} \mu_{B}^{2} S(S+1) /$ $3 k_{B} P_{m}=0.0332 \mathrm{~cm}^{3} \mathrm{~K} / \mathrm{g}$, where $S=12, g=2$, and the molecular weight $P_{m}=2347.06$. The positive $\theta$ confirms the ferromagnetic nature of the ordered phase. From the mean-field equation $\theta=2 z J_{\text {eff }} S(S+1) / 3 k_{B}$, we estimate the effective intercluster magnetic interaction $J_{\text {eff }} \approx$ $1.6 \times 10^{-4} \mathrm{~K}$, and the associated effective field $H_{\text {eff }}=$ $2 z J_{\text {eff }} S / g \mu_{B}=3.5 \times 10^{2}$ Oe coming from the $z=12$ nearest neighbors.

The maximum value of $\chi^{\prime}$ is seen to vary only weakly with $\omega$, which we attribute to the anisotropy. The total activation energy of $\mathrm{Mn}_{6}$ amounts to $D S^{2} \approx 1.5 \mathrm{~K}$; i.e., it is about 45 times smaller than for $\mathrm{Mn}_{12}$. Accordingly, one expects the superparamagnetic blocking of the $\mathrm{Mn}_{6}$ spins to occur when $T \simeq T_{B}\left(\mathrm{Mn}_{12}\right) / 45$, that is, below $\simeq 0.12 \mathrm{~K}$. In other words, for $T \rightarrow T_{c}$, the approach to equilibrium begins to be hindered by the anisotropy of the individual molecular spins. We stress, however, that the frequency dependence of $\chi^{\prime}$ observed here is very different from that of the well-known anisotropic superparamagnetic clusters or that of spin glasses. Below $T_{c}, \chi^{\prime}$ decreases rapidly, as expected for an anisotropic ferromagnet in which the domain-wall motions become progressively pinned. The associated domain-wall losses should then lead to a frequency dependent maximum around $T_{c}$ in the imaginary part, $\chi^{\prime \prime}$, as seen experimentally. Indeed, although 
the $\mathrm{Mn}_{6}$ spins can be considered as nearly isotropic at high temperatures, the anisotropy energy $(\simeq 2 D S)$ is of the same order as the dipolar interaction energy $\mu^{2} / r^{3} \simeq 0.1 \mathrm{~K}$ between nearest neighbor molecules. Thus the ordering should be that of an Ising dipolar ferromagnet.

Additional evidence for the ferromagnetic transition at $T_{c}$ is provided by the electronic specific heat $c_{e}$ [11], shown in Fig. 3, which reveals a sharp peak at $0.15(2) \mathrm{K}$. Numerical integration of $c_{e} / T$ between 0.08 and $4 \mathrm{~K}$ gives a total entropy change of about $3.4 k_{B}$ per molecule, very close to the value for a fully split $S=12$ spin multiplet (i.e., $k_{B} \ln (2 S+1)=3.22 k_{B}$ ). We thus may attribute the peak to the long-range order of the molecular spins. We note, however, that at $T_{c}$ the entropy amounts to about $1 k_{B}$ per spin, showing that only the lowest energy spin states take part in the magnetic ordering.

We have performed Monte Carlo (MC) simulations for an $S=12$ Ising model of magnetic dipoles on an orthorhombic lattice with axes $a_{x}=15.7 \AA, a_{y}=23.33 \AA$, and $a_{z}=16.7 \AA$, which approximates the crystal structure of $\mathrm{Mn}_{6}$. The model includes dipolar interactions as well as the anisotropy term $-D S_{z}^{2}$ given in Eq. (1). Neglecting interactions, the ZFS of the $S=12$ multiplet produced by this crystal field term lead to a Schottky anomaly in $c_{e}$, as shown by the dotted curve in Fig. 3. The fit in the range above $0.5 \mathrm{~K}$ yields $D / k_{B} \simeq 0.013 \mathrm{~K}$, in good agreement with our previous estimates. The intermolecular dipolar interactions remove the remaining degeneracy of the $| \pm m\rangle$ spin doublets. The MC simulations show that the ground state is ferromagnetically ordered, as observed, and predict a shape for $c_{e}$ that is in very good agreement with the experiment. In Fig. 3, we show $c_{e}$ calculated assuming all molecular easy $(z)$ axes to point along $a_{z}$,

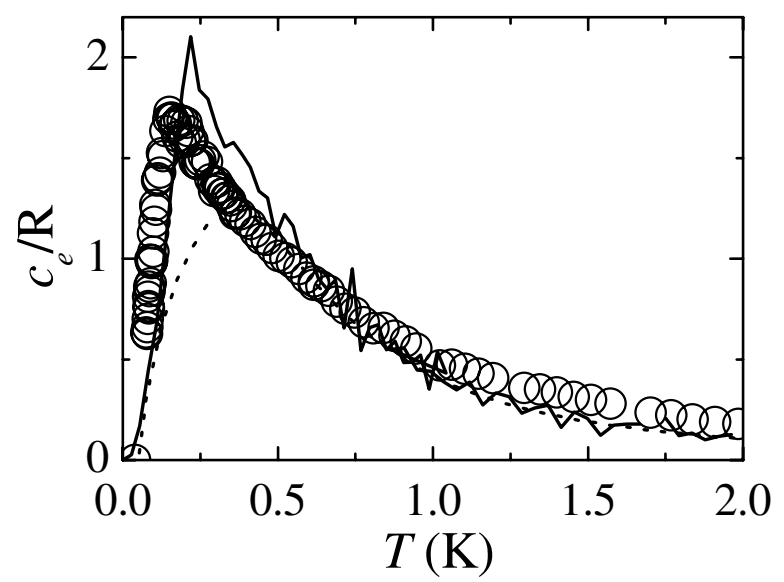

FIG. 3. Electronic specific heat data ( $\bigcirc$ ) of $\mathrm{Mn}_{6}$ at zero field. The dotted line is the Schottky anomaly calculated with $D=$ $0.013 \mathrm{~K}$. The full line is the Monte Carlo (MC) calculation for an orthorhombic lattice of 1024 Ising spins with periodic boundary conditions. For each point, we performed $2 \times 10^{4}$ MC steps per spin. i.e., one of the two nearly equivalent short axes of the actual lattice. Similar results were obtained for other orientations chosen for the anisotropy $(z)$ axis. We note that the Ising simulations give $T_{c}=0.22 \mathrm{~K}$, which is slightly higher than the experimental $T_{c}=0.161(2) \mathrm{K}$. This difference may be related to the finite value of the anisotropy. Model calculations for this crystal structure, assuming classical anisotropic Heisenberg spins with varying anisotropy [12], show that different ferromagnetic ground states are possible, depending on the competition between local crystal field effects and long-range dipolar interactions. The variation of $T_{c}$ with anisotropy, as well as the form of the calculated and observed specific heat anomaly, are specific for dipolar interactions, and differ widely from the analogs for the usual superexchange ferromagnets [13].

We next turn to the specific heat data obtained in varying magnetic field $B$, plotted in Fig. 4 . Even for the lowest $B$ value, the ordering anomaly is fully suppressed, as expected for a ferromagnet [13]. Accordingly, we may account for these data with the Hamiltonian (1) neglecting dipolar interactions. The Zeeman term splits the otherwise degenerate $| \pm m\rangle$ doublets, and already for $B \sim 0.5 \mathrm{~T}$ the level splittings become predominantly determined by $B$, so that the anisotropy term can also be neglected. As seen in Fig. 4, the calculations performed with $D=0$ reproduce the data quite satisfactorily at higher temperatures (dotted curves).

However, when the maxima of the Schottky anomalies are shifted to higher $T$ by increasing $B$, an additional contribution at low $T$ is revealed. It is most clearly visible

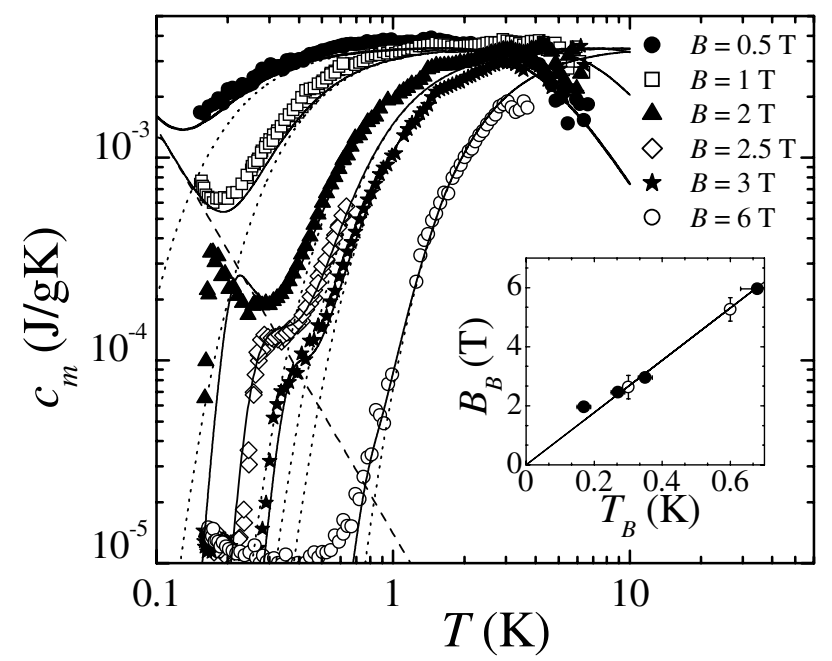

FIG. 4. Magnetic specific heat of $\mathrm{Mn}_{6}$ for various applied fields. Dotted and dashed lines: calculated equilibrium specific heats of the electronic and the nuclear spins, respectively. Full lines: Time-dependent total specific heat calculated taking into account the nuclear spin-lattice relaxation. Inset: Magnetic field $B_{B}$ needed to take the nuclear spins off-equilibrium. $\circ$, obtained from $c_{\text {nucl }}$ vs $B$ isotherms; $\bullet$, from $c_{\text {nucl }}$ vs $T$ at constant field. 
in the curves for $1 \mathrm{~T}<B<2.5 \mathrm{~T}$, and varies with temperature as $c T^{2} / R=4 \times 10^{-3}$. We attribute this to the high-temperature tail of the specific heat contribution $c_{\text {nucl }}$ arising from the Mn nuclear spins $(I=5 / 2)$, whose energy levels are split by the hyperfine interaction with the $\mathrm{Mn}^{3+}$ electronic spins $s$. This interaction can be approximated by $\mathcal{H}_{\mathrm{hf}}=s A_{\mathrm{hf}} m_{I}$, where $A_{\mathrm{hf}}$ is the hyperfine constant and $m_{I}$ is the projection of the nuclear spin along the electronic spin. At high temperatures (i.e., when $\left.A_{\mathrm{hf}} s \ll k_{B} T\right) \quad c_{\text {nucl }} / R \simeq \frac{1}{3} A_{\mathrm{hf}}^{2} s^{2} I(I+1) T^{-2}$ [14]. Taking $A_{\mathrm{hf}}=7.6 \mathrm{mK}$ as used previously to simulate ESR spectra measured on a $\mathrm{Mn}_{4}$ cluster [15], we obtain the dashed line of Fig. 4. This contribution was subtracted from the zerofield data shown in Fig. 3.

A remarkable feature of the experimental data that is not reproduced by these calculations is that, at the lowest $T$, the nuclear specific heat drops abruptly to about $10^{-5} \mathrm{~J} / \mathrm{g} \mathrm{K}$. The temperature $T_{B}$ where the drop occurs depends on $B$ but also on the characteristic time constant $\tau_{e}$ of our (time-dependent) specific heat experiment: the deviation from the (calculated) equilibrium specific heat is found at a lower $T$ when the system is given more time to relax. We conclude that the drop indicates that nuclear spins can no longer reach thermal equilibrium within time $\tau_{e}$. We may write $c_{\text {nucl }}\left(\tau_{e}\right)=c_{\text {nucl }}^{\text {eq }}[1-$ $\left.\exp \left(-\tau_{e} / T_{1}\right)\right]$, showing that the transition should occur when the nuclear spin-lattice relaxation time $T_{1}$ becomes of the order of $\tau_{e}$. These transitions to nonequilibrium provide therefore direct information on the temperature and field dependence of $T_{1}$, which can be related to the fluctuation of the transverse hyperfine field, as produced by the phonon-induced transitions between different levels of the electronic spin [16].

At low $T$ and high $B$, only the ground and the first excited states, $m=+12$ and $m=+11$, need be considered. We may write $1 / T_{1} \simeq\left(1 / \tau_{0}\right) \exp \left\{-\left[g \mu_{B} B\right] / k_{B} T\right\}$, where $1 / \tau_{0}$ plays the role of an attempted frequency for the electron-spin transitions. Clearly, the nuclear spins can be taken out of equilibrium either by decreasing $T$ down to $T_{B}$ at constant field (as in Fig. 4) or by increasing $B$ up to a given value $B_{B}$ at constant $T$. This is indeed observed experimentally (not shown). The effect of the field is just to polarize the electronic spins, which reduces the fluctuations of the hyperfine field, thus effectively disconnecting nuclear spins from the lattice. For a given $\tau_{e}, B_{B}$ must increase linearly with $T_{B}$, which is confirmed by the experimental data plotted in the inset of Fig. 4. The slope gives $\tau_{0} \approx 3 \times 10^{-4} \mathrm{~s}$. Using this value, we have calculated the time-dependent $c_{\text {nucl }}$, shown as the full lines in Fig. 4, and seen to be in reasonable agreement with the experimental data at all $T$ and $B$.

The authors have enjoyed illuminating discussions with Dr. E. Palacios, and Professor P.C. E. Stamp. This work is part of the research program of the "Stichting voor Fundamenteel Onderzoek der Materie" (FOM). F. L. acknowledges a TMR grant from the European Union. J.F. F. acknowledges Grant No. BMF2000-0622 from DGESIC of Spain.

*To whom all correspondence should be addressed. Email address: dejongh@phys.leidenuniv.nl

[1] S. J. White, M. R. Roser, J. Xu, J.T. van Noordaa, and L. R. Corruccini, Phys. Rev. Lett. 71, 3553 (1993).

[2] See, e.g., D. Bitko, T. F. Rosenbaum, and G. Aeppli, Phys. Rev. Lett. 77, 940 (1996); G. Mennenga, L. J. de Jongh, and W. J. Huiskamp, J. Magn. Magn. Mater. 44, 59 (1984).

[3] J. F. Fernández and J. J. Alonso, Phys. Rev. B 62, 53 (2000); J. F. Fernández, Phys. Rev. B 66, 064423 (2002).

[4] C. Sangregorio, T. Ohm, C. Paulsen, R. Sessoli, and D. Gatteschi, Phys. Rev. Lett. 78, 4645 (1997).

[5] S. M. Aubin, N. R. Dilley, L. Pardi, J. Krzystek, M.W. Wemple, L.-C. Brunel, M. B. Maple, G. Christou, and D. N. Hendrickson, J. Am. Chem. Soc. 120, 4991 (1998).

[6] L. Thomas, A. Caneschi, and B. Barbara, Phys. Rev. Lett. 83, 2398 (1999).

[7] F. L. Mettes, F. Luis, and L. J. de Jongh, Phys. Rev. B 64, 174411 (2001); F. Luis, F. L. Mettes, J. Tejada, D. Gatteschi, and L. J. de Jongh, Phys. Rev. Lett. 85, 4377 (2000); F. L. Mettes, G. Aromí, F. Luis, M. Evangelisti, G. Christou, D. Hendrickson, and L. J. de Jongh, Polyhedron 20, 1459 (2001).

[8] G. Aromí, M. J. Knapp. J.-P. Claude, J. C. Huffman, D. N. Hendrickson, and G. Christou, J. Am. Chem. Soc. 121, 5489 (1999).

[9] We have used the crystallographic data for a related complex $\mathrm{Mn}_{6} \mathrm{O}_{4} \mathrm{Cl}_{4}\left(\mathrm{Et}_{2} \mathrm{dbm}\right)_{6}$ [8], in which the $\mathrm{Br}^{-}$ ions are replaced by $\mathrm{Cl}^{-}$.

[10] A. Bencini and D. Gatteschi, EPR of Exchange Coupled Systems (Springer-Verlag, Berlin and Heidelberg, 1990).

[11] The electronic specific heat was obtained by subtracting from the total specific heat the contribution of the lattice, which follows the well-known Debye approximation for low temperatures $c_{\text {latt }} \propto\left(T / \Theta_{D}\right)^{3}$, where $\Theta_{D} \simeq 48 \mathrm{~K}$, as well as the contribution $c_{\text {nucl }}$ arising from the nuclear spins, as discussed in the text.

[12] J. F. Fernández and F. Luis (unpublished).

[13] See, e.g., L. J. de Jongh and A. R. Miedema, Adv. Phys. 50, 947 (2001).

[14] A. Abragam and A. Bleaney, Electron Paramagnetic Resonance of Transition Ions (Clarendon Press, Oxford, 1970).

[15] M. Zheng and G. C. Dismukes, Inorg. Chem. 35, 3307 (1996).

[16] A. Abragam, The Principles of Nuclear Magnetism (Clarendon Press, Oxford, 1961). For a recent application of the theory to $\mathrm{Mn}_{12}$, see Y. Furukawa et al., Phys. Rev. B 64, 104401 (2001). 